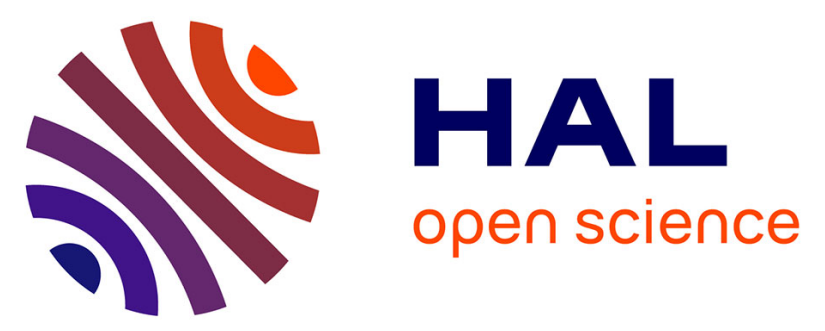

\title{
La distance sociale dans le travail de terrain: compétence stratégique et compétence culturelle dans l'interaction d'enquête
}

François Bonnet

\section{- To cite this version:}

François Bonnet. La distance sociale dans le travail de terrain : compétence stratégique et compétence culturelle dans l'interaction d'enquête. Genèses. Sciences sociales et histoire, 2008, 73, pp.57-74. halshs-00879311

\author{
HAL Id: halshs-00879311 \\ https://shs.hal.science/halshs-00879311
}

Submitted on 4 Nov 2013

HAL is a multi-disciplinary open access archive for the deposit and dissemination of scientific research documents, whether they are published or not. The documents may come from teaching and research institutions in France or abroad, or from public or private research centers.
L'archive ouverte pluridisciplinaire HAL, est destinée au dépôt et à la diffusion de documents scientifiques de niveau recherche, publiés ou non, émanant des établissements d'enseignement et de recherche français ou étrangers, des laboratoires publics ou privés. 
François Bonnet, "La distance sociale dans le travail de terrain : compétence stratégique et compétence culturelle dans l'interaction d'enquête", Genèses, n 73 , 2008, pp. 57-74

\section{La distance sociale dans le travail de terrain : compétence stratégique et compétence culturelle dans l'interaction d'enquête}

"Réduire la distance » entre enquêteur et enquêté apparaît comme un impératif catégorique de l'ethnographie, dans la mesure où l'étrangeté et l'inégalité qui constituent cette distance sont des obstacles méthodologiques bien connus. L'article porte un regard critique sur la notion de distance sociale, en s'appuyant sur un travail de terrain effectué dans un ghetto noir de Brooklyn. La distance se construit dans l'interaction entre enquêteur et enquêté; l'enquêté peut faire preuve de compétence stratégique pour réduire ou instrumentaliser cette distance, tandis que l'enquêteur peut faire preuve de compétence culturelle pour en minimiser les aspects négatifs. L'analyse réflexive des interactions d'enquête n'est donc pas seulement un exercice méthodologique, mais un moyen de produire des données.

François Bonnet est chercheur postdoctorant à l'Observatoire Français des Conjonctures Économiques et travaille sur les questions urbaines et pénales. Sa thèse (cotutelle Sciences Po / Université de Milan) a obtenu le prix Gabriel Tarde de l'Association Française de Criminologie. Il est membre du Center for Urban Research and Policy de Columbia University et enseigne à Sciences Po. Il a publié «Un crime sans déviance : le vol en interne comme activité routinière » dans la Revue française de sociologie (vol. 49, $\mathrm{n}^{\circ} 2,2008$ ). 


\section{La distance sociale dans le travail de terrain : compétence stratégique et compétence culturelle dans l'interaction d'enquête ${ }^{1}$}

"Réduire la distance » entre enquêteur et enquêté apparaît comme un impératif catégorique de l'ethnographie, dans la mesure où l'étrangeté et l'inégalité qui constituent cette distance sont des obstacles méthodologiques bien connus. L'article porte un regard critique sur la notion de distance sociale, en s'appuyant sur un travail de terrain effectué dans un ghetto noir de Brooklyn. La distance se construit dans l'interaction entre enquêteur et enquêté; l'enquêté peut faire preuve de compétence stratégique pour réduire ou instrumentaliser cette distance, tandis que l'enquêteur peut faire preuve de compétence culturelle pour en minimiser les aspects négatifs. L'analyse réflexive des interactions d'enquête n'est donc pas seulement un exercice méthodologique, mais un moyen de produire des données.

\section{Les recherches dans le ghetto et le problème de la distance sociale}

Les dernières années ont vu aux Etats-Unis un regain d'études ethnographiques urbaines dans plusieurs domaines: le ghetto noir-américain (Wacquant 2002a, Anderson 1999, Venkatesh 2000 et 2006), les quartiers latino (Bourgois 1996, Small 2004, Smith 2006, Marwell 2007), les sans-abris noirs (Duneier 1999), les travailleurs pauvres de Harlem (Newman 2000) ou la classe moyenne noire (Pattillo-McCoy 1999, Gregory 1998, Pattillo 2007). Ces recherches nous ont permis de mieux connaître les habitants et les problèmes des villes américaines. Elles ont nourri de multiples débats dont l'un porte sur la question de la relation entre le statut de l'interviewé et celui du chercheur dans le travail de terrain. Cette question est souvent traitée à travers la problématique de la distance sociale entre enquêteur et enquêté. La notion de distance sociale renvoie à l'idée d'un espace social structuré par des caractéristiques comme l'âge, le genre, l'origine ethnique et le statut social. Dans l'interaction d'enquête, la distance sociale a deux dimensions : l'étrangeté et l'inégalité. L'étrangeté renvoie aux problématiques de l'accès au terrain, comme l'incommunicabilité des mondes vécus et les difficultés à nouer la confiance. L'inégalité implique un rapport de pouvoir et donc des conséquences potentiellement négatives de l'interaction d'enquête pour les enquêtés. Les problèmes éthiques de l'ethnographie découlent de cette dimension inégalitaire. De fait, la question de la distance sociale est au principe d'une controverse de l'ethnographie urbaine entre deux de ses meilleurs représentants, Loïc Wacquant et Mitchell Duneier².

\footnotetext{
${ }^{1}$ Je remercie Sudhir Venkatesh, qui a initié cette recherche et l'accompagne intellectuellement, ainsi que Clément Théry, Hugo Bertillot, Alexandre Lambelet et Patrick Le Galès. Je remercie aussi Loïc Wacquant, qui, sans nécessairement approuver tous les arguments développés, a suggéré des modifications pertinentes.

2 Dans un long review essay (plus de 60 pages) commandité par l'American Journal of Sociology, Wacquant recense trois ouvrages : Newman (2000), Anderson (2000) et Duneier (1999). Il leur reproche respectivement sur un ton polémique un compte-rendu moralisant, binaire et aseptisé de leur objet. A la suite de l'article, l'AJS publie les réponses des trois auteurs incriminés qui dénoncent des procédés malveillants et indigne de l'échange universitaire : citations tronquées, « oublis », etc.. L'article de Duneier (2006) s'inscrit dans la continuation de cette polémique. Mon propos n'est ici pas de prendre parti pour l'un ou pour l'autre ; j'estime cependant que Wacquant a
} 
Dans le premier temps de cette controverse, Wacquant (2002b) reproche à Duneier (1999) de montrer ses sans-abris plus vertueux qu'ils ne peuvent raisonnablement l'être. Il pointe la tentation des ethnographes de représenter les sujets sous un jour avantageux, d'occulter certains aspects embarrassants du terrain pour donner une image plus présentable des interviewés, et de vouloir « défendre » des individus auxquels le chercheur s'est progressivement identifié. La propension des sociologues à vouloir représenter de façon positive leurs enquêtés est l'un des problèmes chroniques de l'ethnographie ; Katz (1997) avait par exemple recommandé aux sociologues de ne pas détourner pudiquement le regard quand ils observent des immigrés clandestins frauder cyniquement l'aide sociale, sous prétexte qu'il s'agit d'un préjugé largement répandu et qu'il ne serait pas politiquement opportun de le conforter. Dans un second temps de la controverse, Duneier (2006) dénonce la «naïveté »de Wacquant (2002a), qui a travaillé avec des boxeurs du ghetto de Chicago. Duneier juge parfaitement irréaliste l'idée selon laquelle Wacquant aurait été capable d'abolir la frontière raciale entre lui, universitaire blanc, et ses sujets, des jeunes sous-prolétaires noirs ${ }^{3}$. Duneier appuie son raisonnement sur la remarque célèbre de Liebow (1967) selon qui il existe entre le chercheur et ses enquêtés un «grillage en fer » (chain linked fence).

Ce débat illustre les deux aspects de la distance sociale : l'occultation des faits embarrassants par Duneier (selon Wacquant) se fait au nom du respect des enquêtés, du fait de l'inquiétude éthique qui découle de l'inégalité de position entre Duneier et les sans-abri ; la prétention à l'abolition de la frontière raciale par Wacquant (selon Duneier) renvoie à la question de l'étrangeté et de l'accès au terrain. Chacune de ces deux critiques s'inscrit dans l'histoire de longue durée de l'anthropologie, laquelle suggère deux tendances sur l'évolution de la question de la distance sociale entre le chercheur et son terrain: d'une part, l'idée que la réduction de la distance (comme étrangeté) entre le chercheur et son terrain est la clé de la compétence méthodologique, et d'autre part, une inquiétude croissante sur les risques éthiques potentiels de l'ethnographie et une plus grande considération pour le respect des interviewés.

Concernant l'étrangeté et donc la réduction de la distance, l'armchair anthropology de James G. Frazer et Marcel Mauss, fondée sur les récits de voyageurs et de missionnaires, est désormais méprisée, tandis que le travail de terrain de Malinowski, qui a vécu deux ans avec les Trobriandais, est célébré comme la première ethnographie scientifique. Dans cet esprit, Park avait recruté à Chicago des professionnels qu'il avait converti à la sociologie : Nels Anderson était un hobo que Park avait transformé en sociologue des hobos, Frederick Thrasher et Clifford Shaw étaient des contrôleurs judicaires (probation officers) devenus criminologues, et Louis Wirth était un travailleur social de formation, devenu sociologue du ghetto (Emerson 2001). Depuis les années 1970, le souci de

fondamentalement raison de défendre une ethnographie attentive à la complexité et qui ne cherche pas à rendre service aux enquêtés ; en revanche, les ouvrages choisis pour exemplifier ces dérives, ainsi que certains procédés indélicats, ne me semblent pas des plus pertinents.

${ }^{3}$ Wacquant (2005) se défend d'avoir eu cette prétention et pointe le fait que « le livre se ferme sur DeeDee me rappelant que je ne suis qu'un sociologue, que ça suffit, je peux écrire mon livre » (communication personnelle). 
la plupart des ethnographes de comprendre et d'interpréter le sens subjectif des mondes symboliques (Geertz 1973) marque une évolution positive par rapport à l'époque où l'anthropologie se contentait $d$ ' «inventaires de coutumes ». De ce point de vue, minimiser la distance entre l'ethnographe et son terrain est la clé de la qualité du travail ethnographique. Aujourd'hui, de nombreux auteurs estiment que faire partie d'un groupe ethnique ou racial aide à réaliser un travail de terrain auprès de ce groupe ethnique ou racial. Selon Zavella $(1996)^{4}$, les insiders, c'està-dire les sociologues de couleur qui enquêtent dans «leur» communauté (comme Elijah Anderson, Mario Small ou Mary Pattillo), comprennent mieux les subtilités du langage, gagnent la confiance de leurs interlocuteurs plus facilement, et formulent des problématiques plus respectueuses des communautés auxquels ils appartiennent. Dans la sociologie française, cette idée a par exemple été soutenue par Bourdieu (1993), qui préconisait le recrutement d'enquêteurs socialement proches des enquêtés ${ }^{5}$.

Concernant l'inégalité et donc l'inquiétude éthique, il faut se rappeler qu'un évolutionnisme distinguant «sauvages » et «civilisés » présidait aux considérations théoriques des premiers anthropologues comme Morgan, Tylor ou Frazer (Deliège 2006, Béteille 2007). L'anthropologie est à l'origine une science de gouvernement visant à mieux comprendre les «sauvages » (tribus indiennes aux Etats-Unis, pays colonisés en Afrique) (Emerson 2001). A partir des années 1950-1960, la décolonisation a profondément modifié le rapport des ethnographes à leur terrain, entraînant une remise en cause des problématiques bienveillantespaternalistes et le développement d'un relativisme non seulement méthodologique mais aussi perçu comme arme politique contre la domination symbolique de l'Occident. Le «respect des interviewés» est donc devenu un impératif déontologique de la pratique scientifique. En France, Bourdieu a par exemple conçu l'enquête de La misère du monde de manière à « réduire au maximum la violence symbolique qui peut s'exercer à travers [la relation d'enquête] » (1993: 1391), appelant à « une communication "non-violente" » pour l'enquêté.

Mon propos est de porter un regard critique sur la notion de distance sociale en dissociant la problématique de l'étrangeté et celle de l'accès d'une part (la distance n'empêche pas l'analyse - voir Geertz [1983] et Béteille [2007] $]^{6}$ ), et en soutenant que les obligations éthiques sont au mieux remplies en rendant aux interviewés leur compétence stratégique. Pour ce faire, je vais mettre l'accent sur la notion de réflexivité, dans la lignée des travaux de Rabinow (1977) et Venkatesh (2002). Venkatesh recommande d'opérer un retour réflexif sur la production des données, en partant du principe que l'analyse de la relation d'enquête est elle-même instructive. Selon lui (2002: 92), «l'interaction entre

\footnotetext{
${ }^{4}$ Cité in Emerson (2001 : 114).

${ }^{5}$ Bourdieu (1993) utilise des phrases comme : « surmonter la distance sociale» (p. 1396); « Mais tous les procédés et tous les subterfuges que nous avons pu imaginer pour réduire la distance ont leurs limites » (p. 1399) ; «Lorsque rien ne vient neutraliser ou suspendre les effets sociaux de la dissymétrie liés à la distance sociale, on ne peut espérer obtenir des propos aussi peu marqués que possible par les effets de la situation d'enquête (...)» (p. 1399).

${ }^{6}$ Geertz (1983 : 70) écrit par exemple que parvenir à comprendre le monde vécu des enquêtés selon leur point de vue s'apparente plus à « saisir un proverbe, voir une allusion, comprendre une blague » qu'à «parvenir à la communion». Béteille (2007: 126) soutient la nécessité de «multiples points de vue », à la fois d'anthropologues étrangers et d'anthropologues autochtones. L'article de référence sur la question dans la sociologie américaine est Merton (1972).
} 
l'ethnographe et ses informateurs est elle-même potentiellement révélatrice des propriétés locales de la structure sociale et peut être aussi creusée pour éclairer les questions de recherche ». Le questionnement méthodologique ne doit pas se résumer à décrire à quel point on a réussi à «accéder» au terrain. Il est naïf de croire que le chercheur, parce qu'il serait désintéressé et bienveillant, obtient des «données pures» de la part de ses sujets : ces derniers ne sont pas des êtres passifs mais des acteurs intelligents, compétents, qui peuvent manipuler et instrumentaliser le chercheur.

En analysant ma propre relation (de Français ${ }^{7}$, blanc, alors âgé de 26 ans et post-doc à l'université de Columbia) à mon terrain, un ghetto noir situé à la frontière de Brownsville et de East New York dans le borough de Brooklyn (New York) (2), je vais d'abord montrer comment la distance sociale peut se manifester (3). Ensuite, je vais voir en quoi la distance peut être réduite par les interviewés en fonction de leurs intérêts (4), et comment ces interviewés peuvent instrumentaliser la distance sociale (5). Enfin, je défendrai l'idée que cette distance se construit dans l'interaction en fonction des différentes identités mobilisables par les interactants (6).

\section{Une enquête dans un ghetto noir de Brooklyn}

En janvier 2006, j'ai commencé mon terrain à Brownsville et à East New York pour travailler sur les acteurs de la sécurité, les services sociaux et les organisations locales.

\section{Le ghetto: Brownsville / East New York}

Brownsville et East New York ont toujours été des quartiers pauvres et mal famés (voir Pritchett 2002 et Thabit 2003). Au début du siècle, Brownsville était un ghetto juif ; East New York comportait aussi des populations irlandaises et italiennes. A la fin des années 1950, les Noirs ruraux du Sud et les Portoricains ${ }^{8}$ migrèrent en masse vers les grandes villes du nord-est. Les agents immobiliers jouèrent des préjugés et de la ségrégation pour maximiser leurs profits : ils faisaient parader des familles noires dans les quartiers juifs, italiens et irlandais pour effrayer les habitants, qui vendaient leurs logements à la hâte, bien en dessous du prix du marché. Les agents immobiliers construisaient alors des cloisons à l'intérieur des habitations pour louer ou vendre une demeure prévue pour une famille de quatre personnes à trois familles nombreuses noires. Contraints par la ségrégation, les Noirs ne pouvaient se loger à New York en dehors de quelques quartiers. Les agents immobiliers réussissaient des miracles en termes de création de valeur : ils achetaient à vil prix et multipliaient le profit en entassant les Noirs. La municipalité construisit en urgence du logement social pour résoudre la crise immobilière, ce qui ne fit qu'accélérer la formation de

\footnotetext{
${ }^{7}$ Pour une défense de l'idée selon laquelle les Français sont mieux vus que les Blancs américains par les Noirs, voir Wacquant (2005). Une collègue, Eva Rosen (qui connaît bien la France et travaille dans le ghetto), m'avait au contraire prévenu que les Européens sont perçus par les Noirs comme «encore plus blancs» que les Blancs américains. Mon identité de Blanc français (par opposition à blanc américain) ne m'a jamais semblé déterminante, sûrement parce que j'ai réalisé une grande partie du terrain avec une Américaine (blanche).

${ }^{8}$ Les Portoricains, comme les Cubains, peuvent être blancs comme des Européens, de type hispanique, ou noirs.
} 
ghettos noirs homogènes ${ }^{9}$. Au cours des années 1960-1970, la ville de New York, au bord de la banqueroute, laissa les ghettos à l'abandon; les ordures ménagères étaient ramassées toutes les deux semaines et les panneaux de signalisation n'étaient plus remplacés. La crise économique, la hausse du chômage et la drogue achevèrent d'enfoncer Brownsville et East New York dans la misère. La criminalité devint endémique, les deux precincts (commissariats) totalisant plus de meurtres que dans les quartiers de Harlem (Manhattan), du South Bronx ou de Jamaica (Queens). Au début des années 1990, le maire de Boston en visite à East New York déclara: "cela ressemble au début de la fin de la civilisation ». Aujourd'hui, les effets conjugués de l'incarcération de masse et de la gentrification ont transformé ces quartiers et posent des questions nouvelles que je me suis proposé d'explorer.

\section{Le terrain}

Mon terrain a essentiellement consisté à mener une observation participante de l'implantation de Project Together, une ONG spécialisée dans l'aide aux familles de détenus, à East New York. Project Together est une ONG basée à Manhattan qui a un satellite depuis 10 ans dans le quartier latino du Lower East Side. Ce quartier est maintenant en passe d'être complètement gentrifié. Pour poursuivre ses activités, Project Together a décidé d'ouvrir un nouveau satellite dans le ghetto noir de East New York, à Brooklyn. Le travail de Project Together consiste à former des partenariats avec les forces de l'ordre (la police, le bureau du procureur, les agences de libération conditionnelle ${ }^{10}$ ), pour faire bénéficier les détenus de conditions de liberté conditionnelle plus favorables; en échange, les détenus et leurs familles doivent s'impliquer dans un projet de réinsertion. L'idée est de capitaliser sur les relations interpersonnelles au sein des familles pour avoir plus de prises sur des individus au comportement rendu imprévisible par la toxicomanie. Project Together considère que la connaissance du quartier, de ses habitants et de ses acteurs locaux est cruciale pour la réussite du programme. L'association a donc chargé une directrice de projet de monter des partenariats avec les acteurs significatifs à East New York : les forces de l'ordre bien sûr, mais aussi les autres ONG, l'office de logement social, les églises, les associations de locataires, etc. J'ai rencontré la directrice de Project Together et la directrice de projet pour East New York au moment où elles allaient commencer à prendre des contacts avec ces acteurs. Je me suis associé à la directrice de projet, prénommée Leah. Leah devait préparer l'implantation de Project Together à East New York, ce qui impliquait de rencontrer tous les acteurs que je voulais moi-même

\footnotetext{
${ }^{9}$ Dans les premières années, les activistes juifs de gauche étaient alliés aux militants noirs des droits civiques, et pesaient pour que la Municipalité n'oublie pas les ghettos de Brooklyn ; mais un conflit advint entre le syndicat des enseignants (très majoritairement juif) et les conseils de quartier (noirs). Les conseils de quartier voulaient avoir plus de pouvoir de contrôle sur l'enseignement dispensé dans les écoles du ghetto ; le syndicat des enseignants voulait garder ses prérogatives ; il fit grève pendant plus d'un an, ce qui nourrit un ressentiment âpre entre Juifs et Noirs, qui ne s'est pas dissipé. Sur le racisme des Noirs envers les Juifs et vice-versa, voir Perry et White (1986) et West (1994).

${ }^{10}$ Les agences de libération conditionnelle sont Probation et Parole. Probation gère les individus condamnés à de courtes peines afin qu'ils ne viennent pas encombrer les prisons. Parole gère les détenus en fin de peine et «libérés » pour bonne conduite. Dans les deux cas, les conditions de la liberté conditionnelle sont draconiennes: obligation de rencontrer son conseiller toutes les semaines, tests de toxicomanie, couvre-feu à $21 \mathrm{~h}$, interdiction de dormir ailleurs que chez soi, d'avoir un chien, etc.
} 
interviewer. Nous avons donc travaillé ensemble. Concrètement, Leah rencontrait des acteurs et présentait le projet de Project Together, et je l'accompagnais, ce qui me permettait d'observer la façon dont se déroulaient ces rencontres et de poser mes propres questions. Nous discutions beaucoup des entretiens ensemble et échangions nos notes. Leah m'expliquait des aspects culturels et institutionnels spécifiques au contexte américain, et je conseillais Leah sur de nombreux aspects de son travail. Je n'ai pas été payé par Project Together. Je précise que je me suis toujours présenté aux interviewés comme chercheur à Columbia et non comme employé de Project Together. Pour certains, la distinction avait une signification, mais pour la plupart, je pense que j'étais associé à Leah et donc à l'ONG. J'ai pu à la fois réaliser des entretiens et profiter de ma position d'observateur privilégié pour voir comment se forment les partenariats et les conflits entre acteurs organisés. En 8 mois, Leah et moi avons réalisé 70 entretiens et assisté à une vingtaine de réunions publiques (comme des réunions entre la police et les habitants) ou privées. C'est à partir de cette expérience de terrain que je vais aborder la question de la distance sociale.

\section{L'expérience de la distance sociale}

Dans le ghetto, le sociologue sait bien qu'il devra composer avec des interactions où les rapports de race seront embarrassants; il espère aussi que ses interviewés seront chaleureux (souvent, ils le sont). Mais il ne s'attend pas forcément à ce que les habitants qu'il rencontre essaient de le protéger de la dangerosité criminelle du hood.

Fin juillet, nous avons rendez-vous avec une association d'aide aux sans-abris. Nous sommes en retard et Leah décide de prendre un taxi (en fait, un car service d'une compagnie spécialisée, car aucun taxi jaune n'accepterait de se rendre dans le ghetto). Le chauffeur est un latino qui parle mal anglais et qui se repère mal dans le quartier. Nous ne sommes plus qu'à quelques pâtés de maison des locaux de l'association et nous demandons au chauffeur de s'arrêter, nous finirons à pied. Le chauffeur s'affole, «non, non, vous ne pouvez pas vous arrêter ici, c'est trop dangereux!». Nous lui disons qu'on vient tout le temps ici, qu'on connaît bien, mais il insiste, et je crois qu'il veut finir la course pour gagner un ou deux dollars de plus, mais il dit «j'arrête le compteur, je vous y emmène, c'est trop dangereux ».

Fin avril à Brownsville, nous allons dans une espèce de salle des fêtes d'un immeuble d'un project pour assister à une réunion publique entre la police et les habitants du quartier. La réunion est prévue à $19 \mathrm{~h} 30$ et l'endroit est loin de la station de métro. C'est tant mieux, parce qu'il fait beau, que nous allons beaucoup moins souvent à Brownsville qu'à East New York, et que nous n'avons jamais marché dans cette partie-là du quartier, et d'ailleurs nous marchons un peu au hasard au gré des compromis, parce que nous sommes chacun convaincu de savoir quelle est la bonne direction. Nous demandons notre chemin à un homme noir, la trentaine. Il nous jauge une demiseconde et dit : «je vous y emmène en voiture. Je n'ai pas envie qu'il vous arrive quelque chose ». C'est très aimable à lui, mais nous n'aimons pas vraiment l'idée qu'il nous prenne pour de vulgaires crackers apeurés, cette fois on va trouver. Il insiste, et nous ne voulons pas faire comme si nous avions peur de lui et manquer de respect à son hospitalité, bref nous montons dans sa voiture. Il fait 
quelques commentaires comme «il y a des gens ici, vous comprenez », puis nous lui parlons de Project Together. Il se perd plusieurs fois, tourne autour de l'adresse sans arriver à s'approcher, nous lui proposons de finir à pied, mais il tient à nous déposer juste devant le bâtiment.

Le degré d'interprétation le plus direct (pas forcément le moins pertinent) est que certains habitants témoignent tout simplement de bienveillance à l'égard de leur prochain. Cependant, on sait bien que ces mêmes individus ne sont pas bienveillants avec tous ceux qui marchent dans la rue. C'est parce que nous sommes blancs que nous sommes aidés. Dans le cas que je viens d'évoquer (il y en a eu d'autres), nous n'apparaissons pas seulement vulnérables et donc à protéger ; il semble que le second individu qui nous a aidé voulait suggérer qu'il était moins inquiet pour nous que pour la réputation de son quartier si deux Blancs étaient agressés. Dans tous les cas, bienveillance et étrangeté vont de pair : ce n'est pas parce que les interlocuteurs sont bienveillants que nous avons aboli la distance sociale, bien au contraire. Et comme le note Duneier (2006 : 147), « les relations sociales, même les plus chaleureuses, sont toujours compliquées. Il est possible d'avoir d'excellents rapports avec une personne, et même d'être bien intentionnée à son égard, tout en ayant conscience de sa différence et en se demandant si elle ne va pas profiter de vous ».

\section{Une distance négociable : la compétence stratégique de l'interviewé}

La problématique de la distance sociale est trop souvent réduite à celle de l'accès, comme si une trop grande distance structurale rendait la coopération des interviewés impossible. Mais les enquêtés ne se résument pas à leur position structurale : ils sont aussi animés d'une compétence stratégique qui leur fait se demander à quelle condition se montrer coopératif peut leur profiter. Les interviewés ne sont pas des êtres passifs qui attendent qu'un sociologue leur inspire confiance pour ouvrir leur cœur, mais des individus normaux, c'est-à-dire conscients de leur intérêt. Dans les notes de terrain ci-dessous, on va voir que les interviewés sont d'autant plus enclins à se montrer aimables et coopératifs avec nous que nous avons quelque chose qui les intéresse.

Nous sommes invités à la réunion hebdomadaire d'une coalition d'églises, Worldwide United Churches. C'est un samedi matin à $9 \mathrm{~h}$, il fait encore frais et je suis impressionné et nerveux. Comme d'habitude, la réunion a lieu dans une église qui ressemble à une salle des fêtes mal éclairée, mais cette fois, les gens présents ne sont pas spécialement sympathiques; ils ne savent pas qui nous sommes, et c'est une réunion qui n'est pas ouverte au public, et nous sommes exactement ce que nous avons l'air d'être, des secular rich white kids au milieu de Noirs d'âge mûr ; derrière nous, des femmes font la cuisine pour le déjeuner, et je trouve cette division du travail assez Deep South. Le diacre, un homme grand, osseux et pâle, a l'air presque hostile. Leah, qui va devoir parler à l'assemblée, est évidemment encore plus nerveuse que moi. Elle est la première à parler et visiblement, les pasteurs présents sont un peu agacés et veulent savoir pourquoi elle est là. Je suis plein de respect pour elle, tant son auditoire semble hostile. Elle présente Project Together et explique qu'elle va bientôt embaucher le personnel avec lequel elle compte travailler. Très vite, on lui demande combien de gens elle va 
recruter (trois ou quatre). Le diacre, qui est aussi le trésorier de Worldwide United Churches, est franchement froid, je l'appelle «the bad guy» dans mes notes. Il lui demande si le staff sera adapté au quartier: " ne le prenez pas mal, mais vous êtes blancs et c'est un quartier noir ». Leah soupire de soulagement (moi aussi). Elle sourit et répond : «bien sûr, nous recrutons notre staff dans le quartier, par exemple dans le Lower East Side, notre staff à la Bodega est Latino ». Le diacre n'est pas du tout satisfait par cette réponse et dit avec impatience : «très bien madame, les Latinos c'est très bien, mais ici on est Noir ». Leah dissipe le malentendu et assure qu'elle veut recruter dans East New York. Soudain, l'ambiance change complètement : la tension et la nervosité disparaissent, tout le monde souffle et sourit, et le diacre, serein, glisse: «nous vous recommanderons quelques personnes». Les pasteurs applaudissent. Leah revient à sa place, soulagée, nous échangeons quelques mots. Un autre invité vient présenter son programme, une sorte de fête de quartier sur le thème de la santé. Un participant lui demande à la fin : «c'est très bien tout ça, la santé, mais ici nous avons besoin d'emplois, vous pouvez nous aider là-dessus ? ». L'invité ne peut pas. Le président de séance et le diacre se tournent vers Leah avec insistance et le diacre dit : «bon, pour l'emploi, on a quelque chose, la dame ici va faire quelque chose ». Leah se raidit: Project Together n'est pas censé aider les clients à trouver un travail. Elle ajoute, maladroitement: «euh, on pourra peut-être les renvoyer vers le programme Alpha School ». Les pasteurs, le diacre en particulier, sont contrariés. Ils lui reposent deux ou trois fois la question, Leah ne comprend pas qu'ils aient pu comprendre que Project Together allait aider à trouver un emploi. Le diacre dit très abruptement : " madame, vous avez dit tout à l'heure que vous recruteriez dans le quartier». Leah comprend le malentendu et confirme. A la fin de la réunion, le diacre vient nous voir et nous salue chaleureusement.

Il est tentant de céder à une épistémologie selon laquelle le chercheur peut évoluer sur son terrain en pur observateur - une épistémologie paternaliste, puisque les enquêtés sont conçus comme incapables de comprendre la logique des relations sociales. En réalité, à la seconde où le chercheur pose le pied dans le ghetto, ses interviewés savent que celui-ci doit quémander leur coopération, et ils se demandent donc ce qu'il a à offrir et comment ils vont pouvoir en tirer parti.

$\mathrm{Au}$ cours de nos pérégrinations, un nom est devenu mythique à East New York: Brother Mitchell, le chef de Man Up !, une association aux contours incertains (surveillance de la police, encadrement des enfants, défense occasionnelle des hommes dans les affaires de violence conjugale). Brother Mitchell est aussi le principal lieutenant de Charles Barron, un politicien local, ancien Black Panther et connu pour ses diatribes anti-blancs. Brother Mitchell est apparemment très respecté par les jeunes du quartier. Nous le rencontrons lors d'un rassemblement en l'honneur d'une fillette tuée par balle la veille. Il est froid et distant, mais accepte les cartes de visite de Leah et de Pat (une employée noire de Project Together). Trois jours plus tard, il appelle Pat, mentionnant explicitement qu'il l'appelle elle et non Leah parce que Pat est Noire et parce qu'il est toujours méfiant «quand des Caucasiens viennent à Brownsville / East New York sous le prétexte d'aider». Dès cette première conversation, Pat indique que l'association de Brother Mitchell, Man 
Up !, est au plus mal financièrement et que c'est certainement pour cette raison qu'il a contacté Project Together. Deux semaines plus tard, il rencontre Leah, lui présente Man Up! en détail, lui explique qu'il aimerait travailler comme consultant pour Project Together et finit par appeler Leah $«$ Sista Leah ${ }^{11}$.

L'idée selon laquelle la compétence du chercheur réside dans sa capacité à inspirer la confiance n'évoque pas la relation entre des humains réels. Malinowski (1933) a ironisé en son temps sur ce regard condescendant du savant qui traite ses sujets comme des imbéciles incapables de raisonnement stratégique : «Comparez le sauvage en chair et en os, toujours prêt à se soustraire à ses devoirs, vantard et plein de jactance lorsqu'il s'en est acquitté, au mannequin que décrivent les anthropologues, qui suit servilement la coutume et obéit automatiquement à n'importe quelle règle ». Dans le ghetto comme ailleurs, les interviewés sont assez intelligents pour se figurer leur intérêt à coopérer avec les sociologues. Ne pas en être conscient pose problème pour l'analyse des données ; l'occulter sciemment (sous prétexte de dépeindre ses interviewés sous un jour favorable) est une insulte à leur compétence stratégique.

\section{L'instrumentalisation de la distance sociale par les interviewés}

Avant d'être vécue, la distance sociale est d'abord imaginée et fantasmée ; elle commence à exister avant que le terrain ne commence, au sein d'un contexte social particulier. Dans le contexte américain, la distance sociale est avant tout raciale, le rapport de race comprenant à la fois la dimension purement ethnique et la dimension de classe : les Blancs sont aisés, les Noirs pauvres et les Blancs pauvres sont les white trash. Aux Etats-Unis, les Blancs bien élevés ne prononceront jamais le mot nigger (nègre) : ils diront toujours le n-word, même s'il s'agit de relater une conversation tenue par des tiers, même s'il s'agit de s'indigner d'un usage raciste du mot. Le mot nigger ne sort jamais de la bouche d'un Blanc en public. Il dira : «j'ai vu deux jeunes Africains-Americains qui se disaient le n-word aujourd'hui ». Cet article n'a pas vocation à refaire l'histoire de l'esclavage et de la ségrégation, mais il est important de garder à l'esprit que la séparation des races, y compris dans une ville réputée relativement harmonieuse comme New York, est prégnante dans le contexte américain. Les lieux de sociabilité ne sont pas mixtes, les couples mixtes sont mal regardés par les Blancs comme par les Noirs, et les Blancs et les Noirs ne vivent pas dans les mêmes quartiers. Les conversations privées et les forums de discussion anonyme sur Internet fourmillent d'anecdotes relatives au racisme, aux accusations de racisme et aux instrumentalisations des accusations de racisme. Aller faire du terrain dans le ghetto, c'est se jeter dans la gueule du loup des rapports de race; c'est confronter sa mauvaise conscience et sa culpabilité postcoloniale au ressentiment

\footnotetext{
${ }^{11}$ Normalement, les termes de Sister et Brother ne sont utilisés que par les Noirs et pour les Noirs. La plupart du temps, quand un Blanc utilise ces mots, c'est par dérision, voire par intention raciste, comme le fait d'appeler un Native American «Chief ». Se faire appeler Sister ou Brother par un Noir quand on est blanc dans un contexte amical est donc un signe très positif, presque flatteur, comme si on avait prouvé que l'on était vierge de tout racisme. Je me suis moi-même fait appeler «Brother François » un nombre incalculable de fois, notamment par le pasteur qui était persuadé que j'allais écrire un livre à sa gloire.
} 
et à la frustration d'une population qui vit dans la ségrégation et la pauvreté ${ }^{12}$. D'autant plus que les sociologues, même remplis de bons sentiments, ne sont pas les seuls Blancs dans le ghetto : les policiers y sont presque autant détestés que les travailleurs sociaux. La distance sociale se construit ainsi avant de descendre du métro, dans l'anticipation de la méfiance et de la suspicion, une anticipation excitée par la conscience un peu cynique que c'est cette distance même qui est au principe de l'intérêt de l'ethnographie que l'on réalise.

Dans le cadre de l'installation de Project Together à East New York, Leah et moi avons reçu la permission de la part des services sociaux de la mairie d'occuper un bureau dans un de leurs bâtiments. Nous avons donc cohabité avec des travailleurs sociaux (noirs) qui voyaient d'un mauvais œil la privatisation de leur activité, c'est-à-dire le remplacement progressif des travailleurs sociaux municipaux (avec statut de fonctionnaire) par des ONG fonctionnant comme des prestataires de service sur des contrats temporaires (comme le fait Project Together). Dans la première semaine de notre arrivée, les travailleurs sociaux nous ont fait sentir (à Leah et à moi) que nous n'étions pas les bienvenus.

Tous les jours, le repas de midi est un problème, parce que nous marchons beaucoup, nous avons faim, et la nourriture à Brownsville et à East New York est très grasse et de mauvaise qualité. Nous demandons aux travailleurs sociaux où ils vont manger et s'ils nous recommandent un endroit en particulier. Nous n'occupons un de leur bureau que depuis quelques jours et l'ambiance est froide. Au lieu de nous répondre, Margaret nous enjoint de rester sur Sutter Avenue, la rue principale, là où il $\mathrm{y}$ a des commerces et des patrouilles de police, parce que les petites rues sont dangereuses pour nous. Nous sortons en maugréant. Nous allons finalement dans une cafétériabuffet assez loin. Pendant le repas, un homme massif s'énerve contre le caissier et lui jette une canette en fer pleine au visage ; le caissier saigne, l'homme s'en va. J'engage la conversation avec un employé qui fait le ménage ; il parle à peine anglais, en fait il est Burkinabé et nous parlons français. Il m'explique que le client violent n'a pas eu la réduction qu'il escomptait pour une formule et donc n'a pas eu la canette de bière gratuite. Quand nous rentrons au bureau, nous racontons l'histoire aux travailleurs sociaux, principalement pour faire la conversation, et Margaret nous dit de manière condescendante et sarcastique : « et donc vous pensez que c'est typique, c'est ça ?».

Margaret trace la frontière entre eux et nous en nous renvoyant à notre altérité, qu'elle contraste avec sa proximité au monde du ghetto : nous devons faire attention à notre sécurité et nous ne cherchons qu'à confirmer nos préjugés de Blancs sur la violence dans le hood, tandis qu'elle n'a pas besoin de marcher sur les avenues principales, elle sait comment ça se passe à East New York. Margaret nous renvoie à notre identité de rich white kids (Leah a 25 ans, j'en ai 26, tandis que Margaret approche la cinquantaine) qui ne connaissent rien au ghetto et nous ne pouvons rien répondre. Une autre façon de faire sentir au chercheur sa propre étrangeté au monde qu'il essaie de comprendre est de

${ }^{12}$ La notion controversée de liberal guilt (culpabilité de gauche) ou de white guilt (culpabilité des Blancs) est largement utilisée aux Etats-Unis, notamment par les conservateurs. Le sociologue Dalton Conley l'utilise pour parler de sa mère dans un essai autobiographique largement consacré aux relations raciales - l'auteur ayant grandi dans la seule famille blanche des logements sociaux noirs et portoricains du Lower East Side à New York (Conley 2001). 
souligner les propriétés implicites de sa couleur de peau. Être blanc, c'est être riche - une richesse qui s'oppose à la pauvreté qui caractérise les foyers de l'ensemble de logement social d'où proviennent les participants d'une réunion des patrouilles des locataires ${ }^{13}: 61,4 \%$ des habitants vivent en dessous du seuil de pauvreté ${ }^{14}$.

Au début de la réunion des patrouilles de locataires, une femme s'approche de moi pour me vendre des sucreries. Je n'en mange jamais, je refuse machinalement. Elle s'arrête devant moi, avec un air très insistant, et me dis : «c'est pour la cérémonie de remise des diplômes à l'école ». Je le ressens comme si elle disait: 'tu vas te dépêcher de payer, on sait que tu as les moyens, déjà que tu n'as rien à faire ici'. Je me liquéfie de honte et lui achète deux barres chocolatées. Elle fait une petite grimace, que j'interprète comme 'je ne te félicite pas'.

Dans cette courte interaction se jouent toutes les ambiguïtés liées aux rapports de race : mon impair originel entraîne une série de réactions saturées d'implicites raciaux. Je sais qu'elle n'a pas insisté quand les autres participants ont refusé d'acheter ses sucreries ; j'en déduis donc qu'elle insiste parce qu'elle présuppose que j'ai les moyens d'acheter et le devoir moral de le faire, comme si je devais payer ma participation à cette réunion d'une façon ou d'une autre. Mais peut-être suis-je paranoïaque ; peut-être veut-elle seulement maximiser sa recette. Je n'en sais rien, et je ne peux pas le savoir. Sur le coup, sans l'ombre d'une hésitation, j'ai pensé que je me faisais extorquer deux dollars parce que suis blanc, qu'un refus réitéré serait incorrect, qu'un comportement incorrect d'un Blanc envers un Noir est toujours susceptible d'être accusé de racisme et que je ne peux pas me permettre de risquer de me faire accuser de racisme. Au cours de mon enquête, je n'ai pas été visé par une remarque personnelle directe sur ma whiteness; $\mathrm{j}$ 'ai cependant assisté à une réunion publique où une femme politique noire a tenu des propos anti-blancs.

Réunion des locataires de Van Dyke (le complexe de logement social). Lisa Kenner, la présidente de l'association, est très excitée, car elle a prévu une surprise : elle a invité une politicienne, Lenora Fulani ${ }^{15}$, à parler à la fin de la réunion. Lenora Fulani commence son discours par une violente diatribe anti-blancs, et notamment contre « ces Blancs riches qui vont en Europe, vont dans des restaurants huppés et emmènent leurs enfants à leur travail à Wall Street». Je suis interloqué ; c'est la première fois que j'entends des propos anti-blanc à East New York. Je me demande un instant si je ne suis pas en train d'assister à quelque chose que je n'aurais pas du voir. Je regarde Lenora Fulani. Je vois qu'elle fuit mon regard et je me rends compte qu'elle est aussi embêtée que moi, qu'elle n'avait pas

\footnotetext{
${ }^{13}$ Les patrouilles de locataires (tenants patrols) sont des dispositifs mis en œuvre par l'organisme qui gère les logements sociaux; il s'agit de confier aux habitants (souvent des retraités) une partie de la surveillance de l'immeuble.

${ }^{14}$ Le seuil de pauvreté aux Etats-Unis est calculé en fonction des revenus nécessaires à un niveau de vie «tolérable », à la différence du mode de calcul dans l'Union européenne, qui fixe le seuil de pauvreté à $60 \%$ du salaire médian.

${ }^{15}$ Lenora Fulani est un personnage mineur, quoique familier, de la vie politique new-yorkaise. Elle a été la première femme et première Noire à pouvoir se présenter pour l'élection présidentielle dans les 50 états; elle a soutenu Pat Buchanan, généralement considéré comme proche de l'extrême-droite raciste, en 2000 ( sic. Militants noirs et extrémistes racistes ont parfois su trouver des terrains d'entente aux Etats-Unis ; voir Marable [1999].)
} 
prévu que des Blancs puissent être dans la salle, et que sa harangue a l'air un peu vide, puisque tout le monde s'accommode très bien de notre présence.

Pour les politiciens noirs, il n'est pas inhabituel d'appuyer les discours politiques sur l'animosité raciale. L'élu local au conseil municipal, Charles Barron, soutient Robert Mugabe (pour avoir exproprié les fermiers Blancs du Zimbabwe) et affirme que les Blancs conspirent pour cacher au monde que les anciens Egyptiens étaient en fait noirs (le problème étant de savoir si ce sont des Noirs ou des Blancs qui ont construit les pyramides).

Symétriquement, des interviewés noirs m'ont dit des choses qu'aucun Blanc ne dirait publiquement. Par exemple, un pasteur voulait me convaincre qu'il est le mieux placé pour organiser la prestation de services sociaux dans le quartier, parce qu'il est plus au fait des réalités que les travailleurs sociaux. Il m'explique : «Le problème, c'est que quand tu donnes de l'argent à un Noir pauvre, il achète une chaîne $h i-f i »$. Il a ajouté, en référence aux problèmes dans les logements sociaux : «le problème, c'est que la mère est sur le bail, la fille sous-loue, et le baby's daddy ${ }^{16}$ est un parasite. Les filles pensent qu'elles ne sont personne jusqu'à ce qu'elles soient avec un connard qui exploite tout le monde et rackette la pension de la grand-mère. (...) La vérité n'est pas belle. La vérité n'est pas popular. Le problème, ce n'est pas de décrocher un emploi, c'est de le garder. La dernière chose que les gens [les Noirs du ghetto] achètent, c'est la nourriture et les couches $d u$ bébé ». Il ne fait aucun doute que ce pasteur est parfaitement conscient de ce qu'il dit: par exemple, son discours sur les travailleurs sociaux varie grandement selon que je le vois avec Leah (qu'il considère comme une travailleuse sociale) ou non. Il sait que je suis censé m'indigner si je devais entendre les mêmes propos dans un autre contexte. En tenant un discours aussi frontalement provocateur, il sait qu'il retient mon attention bien plus que s'il égrenait la litanie des maux qui frappent les quartiers noirs. Comme Margaret, comme la vendeuse de sucreries à la réunion des locataires, le pasteur utilise un répertoire de références raciales fondé sur une norme antiraciste afin de provoquer un effet sur leur interlocuteur. Mobilisées dans l'interaction, ces références sont efficaces parce que je ne peux pas me soustraire à cette norme, même si j'ai des raisons de penser qu'elle est habilement instrumentalisée.

\section{Distance sociale et compétence culturelle}

Dans les sections précédentes, on a vu qu'une conception de la distance sociale comme attribut était naïve: les interviewés sont capables de jouer la proximité quand c'est leur intérêt, et capables également de jouer sur la peur de leur interlocuteur blanc de paraître raciste pour parvenir à leurs fins. Mais le jeu ne concerne pas que les enquêtés : il relève aussi de la compétence du chercheur de se familiariser avec les codes, les conventions et les coutumes qui régissent le monde qu'il essaie d'enquêter. Maîtriser ces codes permet de neutraliser l'étrangeté et de mobiliser d'autres identités que l'identité raciale. L'enjeu est ici

\footnotetext{
${ }^{16}$ Le concept de baby's daddy et de baby's mama renvoie à la structure particulière de nombreuses familles noires dans le ghetto. Il est tellement fréquent que les jeunes femmes aient des enfants avec des pères différents que les concepts de père, mère, concubin, partenaire n'ont aucun sens. Pour le père biologique de leur enfant, les jeunes femmes disent baby's daddy («le père du bébé »), un terme qui est neutre en ce qui concerne le statut affectif dudit père par rapport à la mère.
} 
de montrer avec Duneier (2006 : 147) que «"se fondre" dans le terrain » (réduire la distance) n'est de toutes façons ni « possible » ni « nécessaire ». En s'appuyant sur une conception de la culture comme «boite à outil » et répertoire pour l'action (Swidler 1986) ${ }^{17}$, on peut comprendre qu'il est tout à fait à la portée du sociologue déterminé à faire son terrain d'acquérir petit à petit des «compétences culturelles » qui lui permettent d'interagir de façon fluide avec ses interlocuteurs.

Par exemple, la première fois que j'ai assisté à une réunion, j'ai omis de répondre au «bonjour » de l'orateur qui commençait son discours. Dans la société blanche, en Europe comme aux Etats-Unis, une personne qui dit bonjour à un public n'attend pas que toute la salle lui réponde en chœur: bonjour ! Dans la société africaine-américaine, si un pasteur, un conférencier, ou n'importe quel plénipotentiaire dit bonjour à son public, tout le public se doit de rendre le bonjour d'une voix forte. Dans une réunion de dix personnes, ne pas dire bonjour (et avoir un regard étonné à la place) se remarque tout de suite : on passe pour un Blanc. Une façon plus grossière d'enfreindre la règle du bonjour est de ne pas marquer soi-même un temps de pause lorsqu'on dit bonjour au début d'une allocution publique. Il en va de même pour le rapport à la religion. Les Africains Américains ont une pratique religieuse très soutenue (Lincoln et Mamiya 1990, PattilloMcCoy 1998) et toute réunion publique commence par une prière. Les participants se lèvent et se tiennent la main, baissent la tête, murmurent des «oh Lord » et des «amen ». Immanquablement, le sociologue blanc, les premières fois, ne comprend rien, est surpris, et sa gaucherie montre son incompétence culturelle. Mais là encore, quelques semaines de pratiques suffisent pour bien connaître les moments où il faut montrer un air grave et pénétré, dire «amen » ou embrasser son voisin. Un mois après ma première réunion publique, je note « je suis maintenant capable de passer toute la prière à regarder mes pieds et à me retenir de scruter les gens. Je dis même 'amen' à la fin ».

Si ne pas rendre le bonjour fait immanquablement perdre de la crédibilité, le rendre alors que ses interlocuteurs ne s'y attendent pas est très positif; de même, se montrer compétent en us religieux est très apprécié. Cela montre que le jeu autour du respect ou non des règles de courtoisie est moins une affaire de normes sociales ou d'essences indépassables qu'une affaire d'épreuves au cours desquelles l'identité de l'intrus est solidifiée. Le Blanc culturellement compétent a toutes latitudes pour faire valoir à ses interlocuteurs noirs d'autres identités - jeune chercheur de Columbia University, Français, habitant de tel quartier, adjoint de la directrice de projet de Project Together, etc. - avec lesquelles un dialogue fructueux est immédiatement possible. Quand Leah et moi rencontrons des Noirs qui travaillent dans le secteur associatif à Bronwsville et à East New York (des collègues de Leah, donc), la dimension « raciale » est absente des entretiens et des discussions et les distinctions de statut se font sur le niveau de prestige de l'ONG à laquelle chacun appartient. En ce sens, la distance n'est pas un attribut, elle se construit dans l'interaction.

\footnotetext{
${ }^{17}$ Swidler (1986) critique l'idée que la culture se réduise aux valeurs qui dirigeraient l'action. Pour elle, « une culture n'est pas un système unifié qui pousse l'action dans une direction cohérente ; c'est plutôt une boite à outils ou un répertoire » (p. 277). Les individus sont donc des « utilisateurs actifs, parfois compétents, de culture » (p. 277). Une culture fournit des ressources pour construire des stratégies d'action.
} 


\section{Conclusion : l'interaction d'enquête comme matériau}

En conclusion, je voudrais revenir sur deux idées. La première, celle que j'ai défendue dans cet article, est que la distance sociale n'est un obstacle que si l'on se cantonne à une conception du chercheur comme enquêteur désintéressé qui recueillerait des informations pures de la part de supports de structures. Etre désintéressé et bienveillant n'a jamais été une condition suffisante pour être bon enquêteur; les données pures sont une illusion positiviste ; les enquêtés sont des acteurs compétents. Il n'est pas nécessaire que les enquêtés nous «acceptent pleinement » ou «nous fassent confiance ». Comme le souligne Duneier (1999: 338-339), nous n'avons-nous même pas cette relation avec nos propres amis, il n'y a donc aucune raison que des interviewés soient dans une relation de pure communication avec nous. La distance sociale n'est pas un attribut fixe des relations enquêteur/enquêté, mais une dimension susceptible de jeu : les enquêtés peuvent travailler à en réduire les effets s'ils y ont intérêt, ou au contraire peuvent en jouer pour renégocier en leur faveur la relation de pouvoir avec leur enquêteur. En ce sens, Wacquant (2002b) a tout fait raison (sur le fond) d'inciter les sociologues à ne pas idéaliser leurs sujets, à ne pas les «romanticiser ». Enfin, l'identité sociale du chercheur ne se réduit pas à sa couleur ou à son statut social, et l'enquêteur comme l'enquêté mobilisent de facto différentes composantes de cette identité dans les interactions. L'important n'est donc pas la distance en ellemême, mais le travail réflexif que le chercheur opère sur son terrain afin de mieux comprendre la logique des relations qui lui ont permis de produire ses données (comme l'ont fait par exemple Rabinow 1977 et Venkatesh 2002).

La deuxième idée que je voudrais défendre est que ce retour réflexif n'a pas qu'un intérêt narcissique : s'intéresser à ces interactions d'enquêtes pour ellesmêmes permet de les transformer en matériau de recherche. Les individus qui voulaient nous protéger dans la rue ne faisaient pas que nous signifier notre identité d'intrus : ils produisaient une description en actes de la stratification sociale du ghetto. Les habitants protecteurs nous défendaient contre les habitants potentiellement menaçants; ils mettaient en scène les divisions du monde social. Ils se présentaient comme des représentants de la population respectable face à la population qui l'est moins, ceux qui travaillent dur et qui vont à l'église versus ceux qui vivent du welfare et du crime. Anderson (1999) a appelé ces deux orientations decent et street, au risque de porter un regard simpliste sur la complexité de la stratification interne du ghetto (Venkatesh 2006) et de réifier le point de vue des decent (Wacquant 2002b). Cette dichotomie est très présente dans la façon dont la strate supérieure des habitants noirs parle de la strate inférieure, et constitue de ce fait un objet d'étude, que le retour réflexif sur le travail de terrain permet d'aborder.

Un autre exemple est celui des pasteurs qui se montraient beaucoup plus affables dès que nous pouvions les mettre en situation de distribuer des emplois à leurs fidèles. Au lieu de s'arrêter à une discussion sur la compétence stratégique des acteurs, on peut se demander: pourquoi des pasteurs dépenseraient-ils du temps et de l'énergie à faire du job placement? Parce que depuis la christianisation des esclaves, les pasteurs jouent le rôle de médiateur et d'intermédiaire entre les Blancs et les Noirs. Historiquement, les pasteurs noirs ont d'abord été utilisés par les planteurs blancs pour contenir le mécontentement des esclaves. Ensuite, ils ont été les seuls représentants de la communauté noire à 
être considérés comme des interlocuteurs valables par la société blanche; et il n'est pas innocent qu'il faille être un homme de foi pour être digne de considération, ce qui illustre s'il le fallait la fracture raciale aux Etats-Unis. Le mouvement pour les droits civiques est né dans les églises noires et son principal animateur était un pasteur baptiste, Martin Luther King. Aujourd'hui, les pasteurs noirs doivent non seulement accomplir leur travail proprement spirituel, mais il leur incombe aussi d'organiser des services sociaux là où l'Etat-providence est défaillant (sur tous ces aspects, voir Lincoln et Mamiya 1990). En somme, porter un regard réflexif sur des interactions d'enquête apparemment anodines peut servir d'entrée à une analyse qui ne se résume pas à de l'introspection. 


\section{Bibliographie}

ANDERSON, Elijah. 1999. Code of the Street: Decency, Violence, and the Moral Life of the Inner City. New York, W.W. Norton

BETEILLE, André. 2007. «Être anthropologue chez soi : un point de vue indien », Genèses, $\mathrm{n}^{\circ} 67$, juin, pp. 113-130

Bourdieu, Pierre. 1993. «Comprendre ». in Pierre Bourdieu (dir.). La misère du monde. Paris, Seuil (Points)

BourgoIS, Philippe. 1996. In Search of Respect. Selling Crack in El Barrio. Cambridge, Cambridge University Press

CONLEY, Dalton. 2001. Honky. New York, Vintage

DELIÈGE, Robert. 2006. Une histoire de l'anthropologie. Ecoles, auteurs, théories. Paris, Seuil

DUNEIER, Mitchell. 1999. Sidewalk. New York, Strauss, Farrar and Giroux

DUNEIER, Mitchell. 2006. "Garder sa tête sur le ring. Sur la négligence théorique et autres écueils de l'ethnographie », Revue française de sociologie, 47 (1), pp. 143-157

EMErSon, Robert M. 2001. Contemporary Field Research. Perspectives and Formulations. Long Grove, Waveland

GEERTZ, Clifford. 1973. «Thick Description. Toward an Interpretative Theory of Culture », The Interpretation of Cultures. Selected Essays. New York, Basic Books

GeERTZ, Clifford. 1983. "From the Native's Point of View": On the Nature of Anthropological Understanding », Local Knowledge. Further Essays in Interpretative Anthropology. New York, Basic Books

Gregory, Steven. 1998. Black Corona: Race and the Politics of Place in an Urban Community. Princeton, Princeton University Press.

KATZ, Jack. 1997. «Ethnography's Warrants », Sociological Methods and Research, 25 (4), May 1997, pp. 391-423

LiEBow, Elliot. 1967. Tally's Corner. A Study of Negro Streetcorner Men. Boston : Little, Brown

Lincoln, C. Eric et Lawrence H. Mamiya. 1990. The Black Church in the African-American Experience. Durham, Duke University Press

MalinowsKi, Bronislaw. 1933. "Le crime et la coutume dans les sociétés primitives », in Mours et coutumes des Mélanésiens, Paris, Payot

MARABLe, Manning. 1999. Black Leadership. Four Great American Leaders and the Struggle for Civil Rights. New York, Penguin

MARWell, Nicole P. 2007. Bargaining for Brooklyn. Community Organizations in the Entrepreunarial City. Chicago, University of Chicago Press

MerTON, Robert K. 1972. "Insiders and Outsiders: A Chapter in the Sociology of Knowledge », American Journal of Sociology, 78 (1), July, pp. 9-47

Newman, Katherine. 2000. No Shame in my Game. The Working Poor in the Inner City. Vintage

PatTillo, Mary. 2007. Black on the Block. The Politics of Race and Class in the City. Chicago, Chicago University Press

PATTILlo-MCCoY, Mary. 1998. «Church Culture as a Strategy of Action in the Black Community », American Sociological Review, vol. 63, December, pp. 767-784

PatTillo-McCoy, Mary. 1999. Black Picket Fences. Privilege and Peril among the Black Middle Class. Chicago, Chicago University Press 
Perry, Huey L. et Ruth B. White. 1986. «The Post-Civil Rights Transformation of the Relationship Between Blacks and Jews in the United States », Phylon, vol. 47, n 1, pp. 51-60

PritcheTt, Wendell. 2002. Brownsville, Brooklyn. Blacks, Jews and the Changing Face of the Ghetto. Chicago, Chicago University Press

RABINOW, Paul. 1977. Reflections on Fieldwork in Morocco. Berkeley, University of California Press (Quantum Book)

SMaLl, Mario Luis. 2004. Villa Victoria: The Transformation of Social Capital in a Boston Barrio. Chicago, University of Chicago Press.

SMith, Robert Courtney. 2006. Mexican New York. Transnational Lives of New York Immigrants. Berkeley and Los Angeles, University of California Press

SWIDLER, Ann. 1986. «Culture in Action : Symbols and Strategies », American Sociological Review, vol. 51, April, pp. 273-286

ThABIT, Walter. 2003. How East New York Became a Ghetto. New York, New York University Press

VenKateSH, Sudhir Alladi. 2000. American Project. The Rise and Fall of a Modern Ghetto. Cambridge, Harvard University Press

VenKATESH, Sudhir Alladi. 2006. Off the Books. The Underground Economy of the Urban Poor. Cambridge, Harvard University Press

VENKATESH, Sudhir. 2002. "Doin' the hustle. Contructing the ethnographer in the American ghetto », Ethnography, 3 (1), pp. 91-111

WEST, Cornell. 1994. Race Matters. New York, Vintage

WACQUANT, Loïc. 2002a. Corps et âme. Carnets ethnographiques d'un apprenti boxeur. Marseille, Agone

WACQUANT, Loïc. 2002b. «Scrutinizing the Streets : Poverty, Morality, and the Pitfalls of Urban Ethnography », American Journal of Sociology, 107 (6), May, pp. 1468-1532

Wacquant, Loïc. 2005. « Carnal Connections: On Embodiment, Apprenticeship, and Membership », Qualitative Sociology, 28 (4), Winter, pp. 445-474

Zavella, Patricia. 1996. "Feminist Insider Dilemmas: Constructing Ethnic Identity with 'Chicana' Informants », in D.L. WoLFE. Feminist Dilemmas in Fieldwork. Boulder, Westview, pp. 138-169 